\title{
Access to intraoperative tumour margin control: a survey of the British Oculoplastic Surgery Society
}

\author{
Huw Oliphant ${ }^{1,2} \cdot$ Tom Oliphant $^{3} \cdot$ Lucy Clarke $^{4} \cdot$ Colin Vize $^{5} \cdot$ Saul Rajak $^{1,2}$
}

Received: 14 May 2019 / Revised: 17 November 2019 / Accepted: 7 December 2019 / Published online: 2 January 2020

(c) The Author(s), under exclusive licence to The Royal College of Ophthalmologists 2020

\begin{abstract}
Background Periocular malignancy is common and in most cases will undergo excision with pre-determined margins and subsequent histological examination. Intraoperative margin control (IOMC) modalities such as fast frozen section (FFS), fast paraffin (FP) and Mohs micrographic surgery (MMS) are being increasingly widely used, though there is a lack of information regarding utility. The aim of this study was to survey members of the British Oculoplastic Surgery Society (BOPSS) to determine attitudes and access to different modalities of IOMC.

Methods A 12-question online survey was disseminated via an e-mail to full members of the BOPSS. The survey was hosted using Qualtrics software via the University of Sussex.

Results The overall response rate was 64 of 165 (38.8\%). MMS was readily available in a neighbouring trust to 23 of 64 respondents (35.9\%). Seven respondents (10.9\%) reported no regional access to MMS. Twenty-nine members had readily available access to FFS (45.3\%) and 37 of 64 to FP $(57.8 \%)$ in their own institution. There is variation in what tumour types would be considered appropriate for IOMC, though most thought clinically ill-defined (morphoeic) basal cell carcinoma (BCC) and squamous cell carcinoma (SCC) should undergo one form of IOMC (90.6\% and $81.3 \%$, respectively).

Conclusion This study highlights variation in availability and utilisation of IOMC amongst oculoplastic surgeons and in different regions of the UK. While the exact place of IOMC in periocular tumour excision is debated, there is a consistent view that it should be available for some tumours. Greater consistency in provision may improve patient outcomes.
\end{abstract}

This research is not under consideration for publication elsewhere. This work was presented at the British Oculoplastic Surgery Society 2019, and the European Society of Ophthalmic Plastic and Reconstructive Surgery 2019.

Huw Oliphant

huw.oliphant@nhs.net

1 Sussex Eye Hospital, Brighton and Sussex University Hospitals NHS Trust, Eastern Road, Brighton BN2 5BF, UK

2 Brighton and Sussex Medical School, Falmer BN1 9PX, UK

3 Department of Dermatology, Royal Victoria Infirmary, Queen Victoria Road, Newcastle Upon Tyne NE1 4LP, UK

4 Newcastle Eye Centre, Royal Victoria Infirmary, Queen Victoria Road, Newcastle Upon Tyne NE1 4LP, UK

5 Hull \& East Yorkshire Eye Hospital, Fountain Street, HU3 2JZ Kingston Upon Hull, UK

\section{Introduction}

Periocular skin cancers are common. In Caucasians, basal cell carcinomas (BCCs) make up around $90 \%$ of periocular malignancies and the remaining $10 \%$ squamous cell carcinoma (SCC), sebaceous gland carcinoma, melanoma and some rarer tumours [1-5]. Excision followed by histological examination is the most widely used treatment modality for periocular tumours [6-10]. Intraoperative margin control (IOMC) in which the tumour and its margins are examined prior to reconstruction is increasingly widely used.

The primary modalities of IOMC are Mohs micrographic surgery (MMS), fast frozen section (FFS) and fast paraffin (FP). MMS uses frozen sections and allows 100\% of the excision margin to be examined. FFS involves freezing the sample and examining vertical sections with a "breadloaf" technique. FP, or rush paraffin assessment, also uses "breadloaf" assessment technique but of fixed tissue that has been embedded in a paraffin block on a shorter preparation cycle, and typically takes around $24 \mathrm{~h}$ to prepare. IOMC offers potentially higher cure rates than 
standard wide margin excision with MMS having the highest cure rates of clearance in both primary and recurrent tumours [10-14]. However, IOMC may prolong the operation and require two stage surgery, increases the cost of the primary procedure and may require patients to travel further or to separate hospitals for the excision and reconstruction.

There is currently little information regarding provision and access to IOMC for patients with periocular malignancy managed by oculoplastic surgeons in the British Isles. This survey aims to assess the provision and utilisation of all forms of IOMC across members of the British Oculoplastic Surgery Society (BOPSS).

\section{Methods}

Over a 3-month period from December 2018 to February 2019, a 12-question survey was disseminated via an e-mail to all 165 full members of BOPSS. Full members of BOPSS are required to be consultants holding certificate of completion of training.

The survey was created using Qualtrics survey software, hosted by the University of Sussex. Response rates were monitored and reminder e-mails were sent out at $\sim 4$ and 8 weeks. Questions were centred upon utilisation and access to the three prior described modalities of IOMC, as well as factors likely to make respondents more likely to consider IOMC. Respondents could also enter free text to report instances where a patient outcome was felt to be impacted by a lack of access to IOMC. The 12 survey questions are seen in Fig. 1.

Participants were encouraged to answer all questions, though it was possible to complete the survey without answering every question. Responses were analysed using the in-built statistical software of Qualtrics.

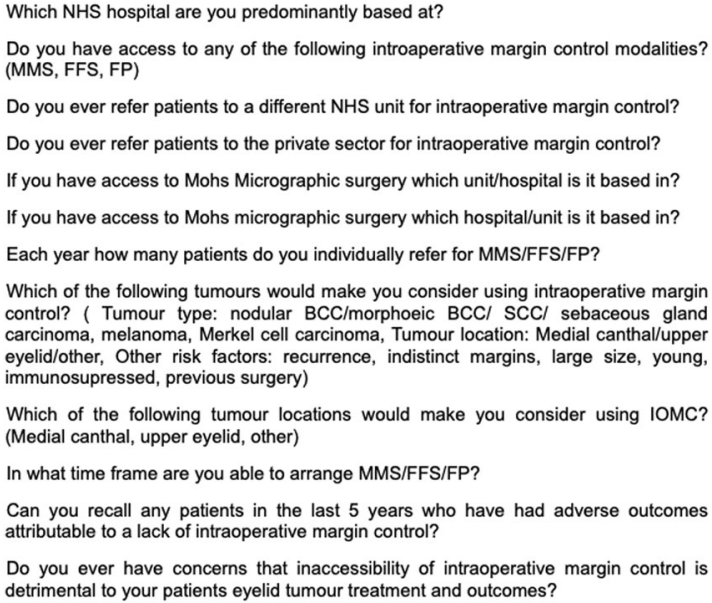

Fig. 1 12-question survey distributed to BOPSS members.

\section{Results}

\section{Demographic data}

The survey was completed by 64 out of 165 (38.8\%) full members of BOPSS. Whilst the total consultant membership of BOPSS is 165 , some members are not in clinical practice, or reside outside the UK, so will not have responded.

Twenty-seven of $46(58.7 \%)$ respondents worked primarily in a university hospital and the remainder in a district general hospital (DGH).

\section{Access to intraoperative margin control}

\section{Mohs micrographic surgery (MMS)}

Fourteen of sixty-four (21.8\%) respondents had MMS in their hospital, with five respondents (7.8\%) reporting restricted access in their own institution. Access was available in a neighbouring trust in 23 of 64 (35.9\%), and 15 respondents $(23.4 \%)$ reported that access was limited in a neighbouring trust. The remaining 7 of $64(10.9 \%)$ had no access to MMS (Fig. 2).

Respondents working within a university hospital setting had access to MMS at the same site as they were based or operated at in $63 \%$ of cases (17 out of 27 responses).

Of those respondents working in a DGH setting and additionally providing information of both their own institution and where MMS is provided, one had access to MMS at the same site.

\section{Fast frozen section (FFS)}

FFS was readily available to 29 of $64(45.3 \%)$ in their own institution, with 21 of $64(32.8 \%)$ having restricted availability at their own trust. One respondent (1.6\%) reported ready access in a neighbouring trust and 2 of $64(3.1 \%)$ limited availability at a neighbouring trust. Six members (9.4\%) did not have any regional access to FFS, and five

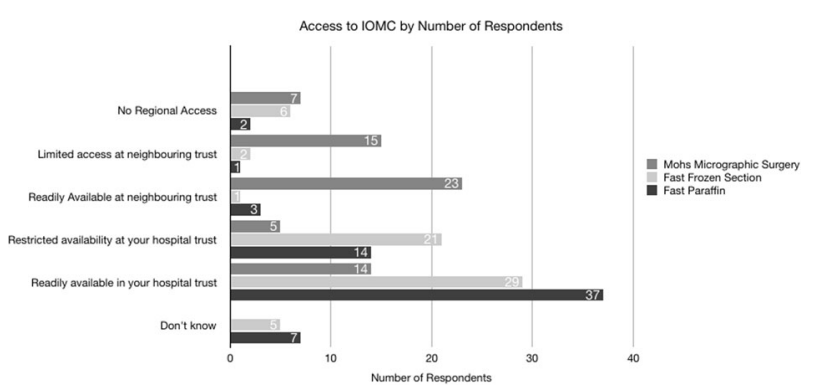

Fig. 2 Access to different forms of IOMC as reported by respondents. 


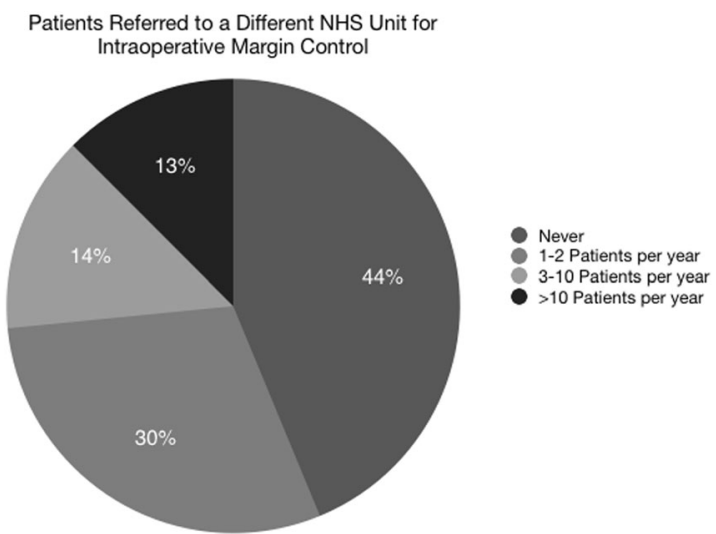

Fig. 3 Percentage of respondents referring to a different NHS unit for IOMC.

respondents $(7.8 \%)$ reported not knowing the degree of access to FFS (Fig. 2).

\section{Fast paraffin (FP)}

FP was readily available to 37 of $64(57.8 \%)$ members in their own trust. Fourteen members (21.9\%) reported restricted access in their own institution. Three out of 64 $(4.7 \%)$ and 1 out of $64(1.6 \%)$ reported ready access and limited access at a neighbouring trust, respectively. Two respondents $(3.1 \%)$ reported no access to FP and seven respondents were not aware of the level of access to FP (Fig. 2).

\section{Referral practice relating to IOMC}

Twenty-eight respondents $(44.0 \%)$ can conduct all their IOMC cases in their own trust; the other thirty-six (56.0\%) refer patients to other hospitals (Fig. 3). Five (7.8\%) respondents routinely refer patients (1-10 patients per year) to the private sector for MMS. Just under half (31/64, $48.4 \%$ ) of the respondents had access to MMS within 1-3 months. However, 9 of $64(14.1 \%)$ had to wait over 6 months for MMS.

MMS was the most widely used IOMC method being used by 45 respondents $(70.3 \%)$. FFS and FP were less widely used with 25 of $64(39.1 \%)$ and 19 of 64 (29.7\%) BOPSS members not using it at all in the last year (Fig. 4).

\section{Indications for IOMC}

\section{Choice of IOMC by tumour type}

58 of 64 respondents $(90.6 \%)$ would consider IOMC for morphoeic/clinically poorly defined BCCs and 27 of 64 (42.2\%) would also consider IOMC for nodular BCC (Fig. 5).

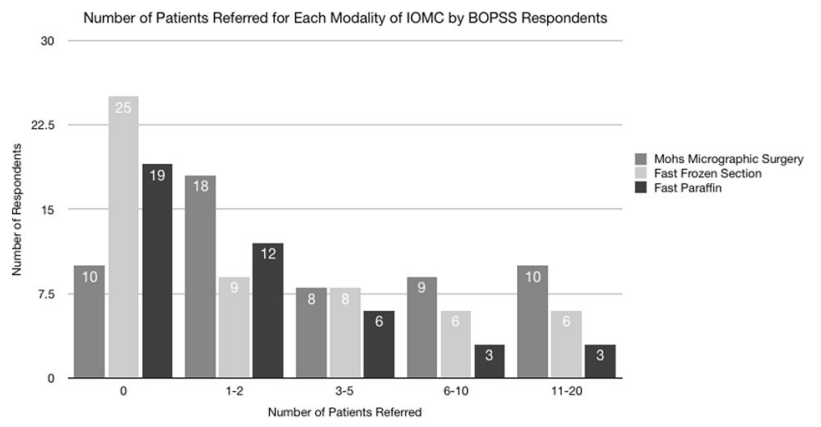

Fig. 4 Number of patients referred personally for each form of intraoperative margin control.

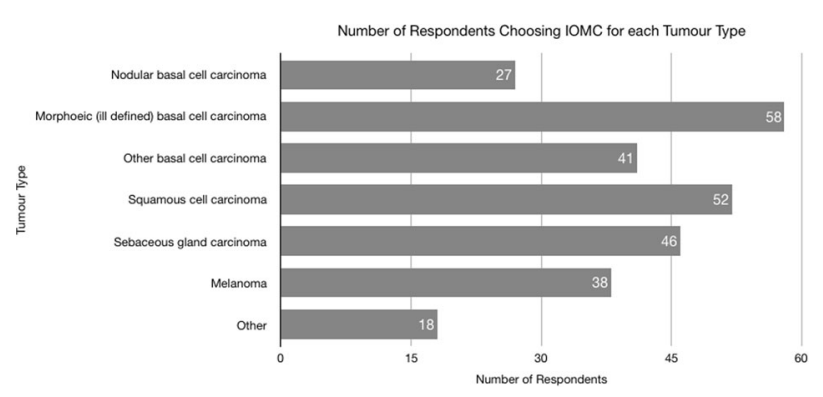

Fig. 5 Tumour types considered for any form of IOMC by BOPSS respondents. (Other tumours included Merkel cell carcinoma, mucinous carcinoma, lentigo maligna and recurrent Bowen's disease).

Fifty-two respondents (81.3\%) would consider using IOMC for SCC, 46 (71.9\%) and 38 (59.4\%) would consider it for sebaceous gland carcinoma and melanoma and a further five participants additionally commented that they would consider IOMC for Merkel cell carcinoma.

\section{Choice of IOMC by tumour location}

Fifty-seven out of 64 respondents (89.1\%) would consider IOMC for a tumour located at the medial canthus and $37(57.8 \%)$ for an upper lid tumour. Three respondents $(4.7 \%)$ considered lateral canthal tumours to be a consideration for IOMC because of the risk of deeper orbital invasion. Eleven BOPSS members (17.2\%) felt any eyelid tumour ought to be considered for IOMC.

\section{Other factors influencing use of IOMC modalities}

Recurrent tumour would be an indication for IOMC for 62 respondents $(96.9 \%)$, indistinct clinical margins for 61 respondents $(95.3 \%)$ and previous surgery or treatment in 52 respondents $(81.3 \%)$. Other indications for IOMC mentioned by respondents included multiple tumours, such as in patients with Gorlin syndrome (nevoid basal cell carcinoma syndrome) and patient 
factors, such as immunosupression, patient choice or whether they are likely not to attend future appointments.

\section{Adverse outcomes and concerns regarding lack of availability of IOMC}

\section{Adverse outcomes attributable to inaccessibility to IOMC}

Seven BOPSS members (10.9\% of responses) reported between 1 and 2 instances of adverse outcomes, and 4 (6.3\% of responses) reported between 3 and 5 cases of adverse outcomes related to a lack of IOMC, which - where further details were given-were typically from incomplete primary SCC excision or medial canthal BCC with orbital invasion.

\section{Concerns regarding inaccessibility to IOMC and patient care}

Nine respondents (14.1\%) did feel that between 1 and 5 patients per year were impacted by a lack of access of IOMC. Two respondents (3.1\%) felt that between 5 and 10 patients were impacted by a lack of access to IOMC and one respondent $(1.6 \%)$ felt that $>20$ patients per year may be impacted by a lack of access to IOMC.

\section{Discussion}

There is a broad consensus amongst BOPSS members that IOMC is indicated for certain BCCs such as those with indistinct clinical margins, and more malignant tumours including SCCs, sebaceous gland carcinoma and melanoma. However, this survey finds that there is wide variation in the availability and utilisation of IOMC.

The risk of recurrence of an eyelid BCC without IOMC ranges from 5 to $100 \%[9,13,15,16]$. Where non-Mohs techniques are used for SCC, 5-year recurrence rates range from 3 to $23 \%$ [17]. Recurrence is affected by numerous factors, including histological sub type, location, tumour size and depth, surgical technique, immune compromise and the presence of perineural invasion for SCC in particular. With margin control modalities such as FFS, the recurrence rate for a primary $\mathrm{BCC}$ at 5 years is $\sim 2-3 \%$. MMS reduces the recurrence rate for primary $\mathrm{BCC}$ at 5 years to between 0 and $1 \%$ and for primary SCC to about $4 \%$ [11, 16-19]. Whilst tumour recurrence rates are demonstrably lower using margin control, and in particular MMS, surgery with IOMC also potentially has disadvantages: it is more time and resource intensive, requiring two and sometimes more procedures, separated by several hours and sometimes days across different hospitals [20].

The precise place for IOMC is debated and varies according to surgeons and patients. It is questionable, however, whether it should vary so widely according to location and availability, particularly given the widespread agreement among BOPSS members that IOMC is indicated for high-risk periocular tumours. Moreover, respondents describe several instances of poor outcomes (orbital invasion) from BCC and SCC excision which may have been avoided by IOMC at primary excision. Hence variation in access to IOMC and with a number of units without access to any form of IOMC and wait of over 6 months for MMS in some locations is of concern and may influence treatment decisions and outcomes.

Provision of IOMC has resource implications for hospital trusts and pathology departments. MMS is a highly specialised service requiring clinician expertise and equipment, and in the United Kingdom it is often undertaken by both a dermatological surgeon who excises and examines the specimen and an oculoplastic surgeon who does the reconstruction. There is a paucity of data on the actual costs of the different methods of histopathological assessment and margin control. However, the tariff devised by the National Institute for Health Research in March 2019 is $£ 1409$ per case of MMS within the National Health Service (NHS) in comparison with around $£ 884$ for standard excision and reconstruction of a periocular BCC, with variation of both according to complexity. One study from the Netherlands has calculated that primary BCCs excised from the facial $\mathrm{H}$ zone using MMS can cost up to $€ 254$ more than standard excision and histological interpretation, although this figure would vary widely according to whether the excision and reconstruction are done by the same or different surgeons and the location and timing of the two stages [21].

There are no current guidelines on the precise indications for the use of IOMC for periocular tumours, although The British Association of Dermatology recommends MMS for those tumours in the periocular region, large tumours (over $2 \mathrm{~cm}$ ), high-risk tumour subtypes, those with poor margins, recurrences and those with perineural or perivascular involvement [22]. Appropriate use criteria (AUC) from the United States of America advocates MMS to be appropriate for all BCCs in area $\mathrm{H}$ (including eyelids) and other highrisk areas [23]. The AUC did, however, note a number of cases $(10 / 69,14.49 \%)$ where MMS was felt to have been used inappropriately. It is therefore important that the relative benefit and resource implications of IOMC are carefully considered for all patients with periocular malignancy.

This study has limitations. Around $60 \%$ of BOPSS members did not complete the survey and there may be differences between responders and non-responders. However, this response rate compares similarly to other major surveys of oculoplastic surgeons. Amongst responders, recall bias may occur particularly with significant events 
such as incomplete tumour and recurrence. In addition, information regarding exact timing of recurrences was not sought in this study which would have offered insight into possible associations between utility of IOMC and poor outcomes. Lastly, this study does not reflect the practice of other clinicians who excise periocular tumours such as general ophthalmologists, plastic surgeons and dermatological surgeons.

This study highlights that despite widespread agreement that IOMC is important for some periocular tumours, there is wide variation in availability and utilisation of IOMC amongst oculoplastic surgeons within the British Isles. MMS is relatively resource intensive and may not be possible for many centres but may be accessible with improvement in patient referral pathways. FFS and FP have logistical challenges but may be cheaper and more widely available. It is beyond the scope of this paper to provide recommendations for which methods of IOMC should be available and when they are utilised, but we would advocate that IOMC is available in all centres in which patients with high-risk tumours may be treated and that there is greater consistency in provision across the health service.

\section{Summary}

\section{What was known before}

- IOMC modalities such as MMS are associated with a high cure rate/low recurrence rate for primary and secondary periocular malignancies.

- There has been little information regarding the availability and utilisation of IOMC by oculoplastic surgeons in the British Isles.

\section{What this study adds}

- There is widespread variation in the access and utilisation of IOMC modalities.

- There is consensus in this survey that high-risk tumours in high-risk locations such as the medial canthus should be treated using one form of IOMC.

- Greater consistency and streamlined referral pathways for IOMC, and MMS in particular, may improve patient outcomes.

\section{Compliance with ethical standards}

Conflict of interest The authors declare that they have no conflict of interest.
Publisher's note Springer Nature remains neutral with regard to jurisdictional claims in published maps and institutional affiliations.

\section{References}

1. Saleh GM, Desai P, Collin JR, Ives A, Jones T, Hussain B. Incidence of eyelid basal cell carcinoma in England: 2000-2010. Br J Ophthalmol. 2016;101:209-12.

2. Lear JT, Tan BB, Smith AG, Bowers W, Jones PW, Heagerty AH, et al. Risk factors for basal cell carcinoma in the UK: case-control study in 806 patients. J R Soc Med. 1997;90:371-4.

3. Margo C, Waltz K. Basal cell carcinoma of the eyelid and periocular skin. Surv Ophthalmol. 1993;38:169-92.

4. Prabhakaran VC, Gupta A, Huilgol SC, Selva D. Basal cell carcinoma of the eyelid. Compr Ophthalmol Update. 2007;8:1-14.

5. Telfer NR, Colver GB, Morton CA. Guidelines for the management of basal cell carcinoma. Br J Dermatol. 2008;159:35-48.

6. Thissen MR, Neumann MH, Schouten LJ. A systematic review of treatment modalities for primary basal cell carcinomas. Arch Dermatol. 1999;135:1177-83.

7. Gulleth Y, Goldberg N, Silverman RP, Gastman BR. What is the best surgical margin for a basal cell carcinoma: a meta-analysis of the literature. Plast Reconstr Surg. 2010;126:1222-31.

8. Trakatelli M, et al. BCC subcomittee of the Guidelines Committee of the European Dermatology Forum. Update of the European guidelines for basal cell carcinoma management. Eur J Dermatol. 2014;24:312-29.

9. Wolf DJ, Zitelli JA. Surgical margins for basal cell carcinoma. Arch Dermatol. 1987;123:340-4.

10. Hoorens I, Batteauw A, Van Maele G, Lapiere K, Boone B, Ongenae K. Mohs micrographic surgery for basal cell carcinoma: evaluation of the indication criteria and predictive factors for extensive subclinical spread. Br J Dermatol. 2016;174: $847-52$.

11. Mohs FE. Micrographic surgery for the microscopically controlled excision of eyelid cancers. Arch Ophthalmol. 1986;104:901-9.

12. Mosterd K, et al. Surgical excision versus Mohs' micrographic surgery for primary and recurrent basal-cell carcinoma of the face: a prospective randomised controlled trial with 5-years' follow-up. Lancet Oncol. 2008;9:1149-56.

13. Rowe DE, Carroll RJ, Day CL Jr. Mohs surgery is the treatment of choice for recurrent (previously treated) basal cell carcinoma. J Dermatol Surg Oncol. 1989;15:424-31.

14. Narayanan K, Hadid $\mathrm{OH}$ and Barnes EA. Mohs micrographic surgery versus surgical excision for periocular basal cell carcinoma. Cochrane Database Syst Rev. 2014:CD007041. https://doi. org/10.1002/14651858.CD007041.pub4.

15. Ho S, Brown L, Bamford M, Sampath R, Burns J. 5 years review of periocular basal cell carcinoma and proposed follow-up protocol. Eye. 2013;27:78-83.

16. Malhotra R, Huilgol SC, Huynh NT, Selva D. The Australian Mohs database: periocular squamous cell carcinoma. Ophthalmology. 2004;111:617-23.

17. Malhotra R, Huilgol SC, Huynh NT, Selva D. The Australian Mohs database, part II: periocular basal cell carcinoma outcome at 5-year follow-up. Ophthalmology. 2004;111:631-6.

18. Conway RM, Themel S, Holbach LM. Surgery for primary basal cell carcinoma including the eyelid margins with intraoperative frozen section control: comparative interventional study with a minimum clinical follow up of 5 years. $\mathrm{Br} \mathrm{J}$ Ophthalmol. 2004;88:236-8.

19. Silverman MK, Kopf AW, Gladstein AH, Bart RS, Grin CM, Levenstein MJ. Recurrence rates of treated basal cell carcinomas. Part 4: X-ray therapy. J Dermatol Surg Oncol. 1992;18:549-54. 
20. Mann J, Al-Niami F, Cooper A, Ghura V. A national survey fo Mohs micrographic surgery in the U.K. $\mathrm{Br} \mathrm{J}$ Dermatol. 2016;174:225-7. https://doi.org/10.1111/bid.14090.

21. Essers BA, Dirksen CD, Nieman FH, Smeets NW, Krekels GA, Prins $\mathrm{MH}$, et al. Cost-effectiveness of Mohs micrographic surgery vs surgical excision for basal cell carcinoma of the face. Arch Dermatol. 2006;142:187-94.

22. Telfer NR, Colver GB, Morton CA, British Association of Dermatologists Guidelines for the management of basal cell carcinoma. Br J Dermatol. 2008;159:35-48. https://doi.org/10. 1111/j.1365-2133.2008.08666.x.

23. Ad Hoc Task Force. et al. AAD/ACMS/ASDSA/ASMS 2012 appropriate use criteria for Mohs micrographic surgery: a report of the American Academy of Dermatology, American College of Mohs Surgery, American Society for Dermatologic Surgery Association, and the American Society for Mohs Surgery. J Am Acad Dermatol. 2012;67:531-50. https://doi.org/10.1016/j.jaad. 2012.06.009. 